\title{
Editorial introduction for Seminars in Immunopathology special issue on "Immune modulation, properties and models of CMV"
}

\author{
Tal Goldberger • Ofer Mandelboim
}

Received: 29 September 2014 / Accepted: 29 September 2014 / Published online: 15 October 2014

(C) Springer-Verlag Berlin Heidelberg 2014

Human cytomegalovirus (HCMV) is a highly clinically relevant pathogen. It efficiently infects large proportion of the adult population around the world, and it establishes lifelong latency. In healthy adults, the virus usually does not cause serious or long-lasting injuries; however, in immunocompromised or immune-suppressed patients (like transplant recipients), infection with HCMV can cause serious illnesses and affect many organs. This is also true in cases of congenital infections. In order to introduce novel antiviral interventions and perhaps even therapeutic agents, a better understanding of the viral life cycle, its tropism, and the ways by which it escapes immune elimination is mandatory. In this special issue of Seminars in Immunopathology, we attempt to establish a multifaceted integrative view of the virus and discuss future implications of our current knowledge.

The effort to introduce effective treatment for congenital HCMV infections is a clinical and scientific challenge, and may advance through novel fetal-maternal interface models

HCMV infections are especially dangerous during pregnancy. Congenital infections can cause intrauterine growth restriction or severe injuries in various body systems in newborns. This makes these infections a highly relevant clinical entity that awaits the introduction of novel and efficient methods of

This article is a contribution to the Special Issue on Immune Modulation, Properties and Models of CMV - Guest Editor: Ofer Mandelboim

T. Goldberger · O. Mandelboim ( $\bowtie)$

The Lautenberg Center of General and Tumor Immunology, The

Hebrew University Hadassah Medical School, IMRIC,

91120 Jerusalem, Israel

e-mail: oferm@ekmd.huji.ac.il therapy. In the review by Weisblum et al., we are introduced to the various aspects of congenital HCMV infections, with special emphasis on the need for "true-to-life" models of the maternal-fetal interface. The long road to successful novel therapies and vaccines begins in the establishment of experimental models that highly resemble the conditions of the human body. However, the species-specificity of HCMV presents an inherent difficulty in the establishment of good animal model. For that reason, much of our knowledge of maternal-fetal interface events during HCMV infection comes from histological analysis of in vivo infected placental tissues and from various in vitro cultures of human cells. These as well as guinea pig and mouse models have contributed to our knowledge, but lack the ability to fully demonstrate the true pathogenesis and kinetics of congenital HCMV infection in humans. A recently established "organ-culture" model, in which human decidual tissues are maintained as a multicelltype organ, allows us to reveal more aspects of the congenital infection, such as cellular tropism and kinetics. It can also serve as a "living" test ground for novel antiviral treatments and interventions.

\section{HCMV-encoded viral Fc $\gamma$ receptors protect infected cells} from immune cells

Knowing the possible serious consequences of HCMV infections, like in the case of congenital infection or infections of immunocompromised patients, we will now zoom in to examine the virus and its pathogenic and stealth promoting elements in the molecular scale. CMV uses significant portions of its vast genome to resist and counteract host immune activation. In a review of $\mathrm{CMV}$-encoded viral $\mathrm{Fc} \gamma$ receptors $(\mathrm{vFc} \gamma \mathrm{R})$ by Corrales-Aguilar et al., we are introduced to a clever immune evasion strategy of CMV that is shared with other famous and clinically relevant pathogens like herpes 
simplex virus, Staphylococcus aureus and Streptococcus sp. The virus expresses its own $\mathrm{vFc} \gamma \mathrm{R}$ proteins, conferring antagonism of the "true" cellular receptors. Different types of Fc $\gamma \mathrm{R}$ are expressed in many different cells of the immune system, and through the binding of the Fc portion (the constant region) of IgG antibodies, trigger various forms of immune activation and depending on the specific receptor, can even reduce immune activation. Four $\mathrm{vFc} \gamma \mathrm{R}$ proteins are now characterized for HCMV, and one is characterized for mouse CMV (MCMV). Interestingly, binding of the Fc portion can be done through a domain which is different than the one bound by endogenous Fc $\gamma$ Rs, as shown for HCMV vFc $\gamma \mathrm{R}$ gp68. Despite that, gp68 was shown to successfully antagonizes various cellular Fc $\gamma$ Rs. Another interesting finding is demonstrated for the MCMV vFc $\gamma \mathrm{R}$ m138 (fcr-1) which was shown to interact not only with IgG but also with NK cell receptor NKG2D and T cell ligands, counteracting immune responses through different pathways. Possible clinical implications and routes of immunotherapy are further discussed in the relevant review.

\section{CMV evades NK cell-mediated elimination by active downregulation of ligands of NK cell receptor NKG2D on infected cells}

Brizic et al. looks at CMV through the murine model. A wide range of MCMV proteins are used to evade NK cell recognition. One notable example is the targeting NKG2D receptor ligands by many MCMV proteins. NKG2D is a killer cell receptor expressed by different immune cell subsets, especially by NK cells. NKG2D, both in humans and in mice, recognizes ligands, also termed "stress ligands." These ligands are upregulated upon cellular stress, infection, and transformation. Interestingly, a significant portion of these ligands are inhibited by two MCMV proteins and in two different cellular compartments, For example, mature H60 molecules are inhibited by MCMV proteins $\mathrm{m} 155$ and $\mathrm{m} 138$ using proteasomal and lysosomal degradation, respectively. HCMV and MCMV also affect the expression of non-NKG2D NK cell ligands such as the unknown ligands for NCR1 (another killer cell receptor expressed by NK cells), PVR, and receptors of the SLAM family. The MHC class I pathway was also shown to be targeted by MCMV, both by downregulation of MHC class I (by m06 and $\mathrm{m} 152$ ) and by the employment of an MHC class I-like decoy molecule (m157) to interact with the inhibitory LY49I murine NK cell receptor. This very much resembles the use of UL18 by HCMV as discussed by Goldberger et al. in the closing review of this issue. Brizic et al. also bring up the recently elucidated phenomenon of NK cell memory. The interaction between Ly49H (another member of the Ly49 family) present on NK cells and the viral MCMV protein $\mathrm{m} 157$ was shown to promote the expansion of Ly49H+ NK cells, thus resembling T cell memory responses.
This process is also associated with the pro-apoptotic factor Bim, and possibly also with DNAM-1 (a co-activating NK cell receptor) signaling. Interestingly, a preferential expansion of NK cells was also observed in humans, as special subset of NK cells $(\mathrm{NKG} 2 \mathrm{C}+$ ) was found to be expanded in HCMV positive patients. Another significant impact of NK cells during MCMV infection is the regulation of T cells. It appears that the effect of Ly49H-m157 interactions during MCMV infection on subsequent $\mathrm{T}$ cell responses is inhibitory, as shown in infections with a virus deleted for $\mathrm{m} 157$. This effect might be attributed to lower antigen loads following NK cell activation. Brizic et al. also discuss interesting possibilities of exploiting MCMV as a vaccine vector, which can be used to immunize against other viruses and possibly even tumor cells.

\section{HCMV shapes the cellular environment to suit its needs and minimize immune elimination through selective post-transcriptional regulation of the cellular proteome and through orchestrated counteraction of immune responses to the virus itself}

The review by Cohen et al. offers a detailed look at the influence of HCMV on the cellular proteome. RNA sequencing is commonly used to characterize gene expression, but we should be aware of miscorrelations between messenger RNA (mRNA) and protein levels in some cases, and specifically membrane surface levels of selected proteins which are prone to post-transcriptional and post-translational modulation. Conclusions from past studies of mRNA expression modulation in HCMV infections show significant changes in the levels of IFN-responsive, cell cycle-related, cell adhesion-related and trascription/translation factor transcripts. Interestingly, few of these mRNAs were found to be modulated by HCMV in the absence of protein synthesis (in the presence of translation inhibitor cycloheximide), including IFN-responsive genes and IFN beta itself. This HCMV-induced rise in IFN-stimulated genes may be attributed to a cellular response to structural features of the virus, specifically the viral envelope glycoprotein B and not to the expression of viral proteins, as discussed by Cohen et al. HCMV uses its proteins to shape the cellular environment to fit its needs, for example, viral protein E86 was shown to induce an increase in mRNA levels of cellular genes that consequently pushes the cell cycle from G0/G1 towards G1/ $\mathrm{S}$. Another study demonstrated an HCMV-induced increase in mitosis-related transcripts which may help shape an "Sphase"-like environment more suitable for the replicating virus. Next-generation sequencing methods further raised the resolution of mRNA surveillance of individual gene transcripts, allowing an almost "real time" view of expression kinetics. In one such study on MCMV, RNA metabolic labeling was used to precisely characterize expression kinetics during the first few hours of infection. An orchestrated rise in 
gene clusters, for example, immune/inflammation-related genes and DNA damage response genes, was demonstrated during early infection. Fascinatingly, these rises were shown to be rapidly counteracted during viral gene expression. The efforts of HCMV to control the cellular environment may be assisted by disruption of intercellular communication. This assumption was examined directly in the protein level and not through mRNA profiling. Indeed, a high-throughput Western blot study demonstrated major effects of HCMV infection on focal adhesion-related and gap junction proteins. A more modern method of protein quantification is mass spectrometry (MS). It was used in one study to demonstrate the presence of angiogenesis and wound healing-promoting factors in the supernatant of HCMV infected cells. In another MS study, a systematic quantitative analysis using tandem mass tags confirmed previous notions of HCMV infectioninduced increase in IFN-stimulated proteins, and the consequent viral counteraction of this cellular response. MS studies were also used to characterize protein-protein interactions for selected HCMV proteins. Given the thorough characterization and profiling of the end-result proteome, it is interesting to know if HCMV infection can modulate protein translation at the level of translation machinery, the ribosome. Indeed, in a recent study that used ribosome profiling, it was shown that HCMV infection not only increases the concentration of small and large ribosomal subunits, but also selectively increases/ decreases the association of specific proteins groups to polysomes. Additional characterization of HCMV-induced cellular changes is further reviewed and includes metabolic, epigenetic, cell-surface protein levels, organelle protein levels, phosphorylation, and lipid profiling.

\section{HCMV uses microRNAs to modulate the cellular environment and evade Killer immune cells}

As demonstrated, HCMV modulates the cellular proteome, both by transcriptional and post-transcriptional regulation. In the review by Goldberger et al., the author illustrates the use of both protein and microRNA (miRNA) effectors to establish this regulation. It is accomplished by inhibition of several important cellular targets by HCMV, some of which are RNA molecules, promoting NK and $\mathrm{T}$ cell evasion, modulating viral replication and controlling the cell cycle. Goldberger et al. discuss human NKG2D and its ligands that are targeted by several herpes viruses (EBV, HCMV, KSHV) and polyomaviruses (BKV, JCV). An interesting phenomenon, implying the importance of the NKG2D system in controlling HCMV infection, is the dual targeting of some of the NKG2D ligands by both proteins and miRNAs. This dual-targeting effort is also seen in an analog fashion for the chemokine RANTES, for the replication controlling BCL2-associated transcription factor 1 and for components of the MHC class I pathway. HCMV's own set of miRNAs are used to modulate various important cellular processes including chemokine synthesis and secretion, replication, and immune-regulating elements like NKG2D ligands and MHC class I pathway components. Some of the HCMV miRNAs show perfect evolutionary conservation in relation to the chimpanzee CMV, implying a pivotal functional role, for example, HCMV-miR-UL112 which functions as a master regulator of viral replication and immune activation. The same important cellular processes are regulated by HCMV in an even more sophisticated way, through the modulation of the cellular miRNA pool. Using miRNA profiling methods, HCMV was shown to induce or reduce the expression of selected cellular miRNAs, to modulate immune activation, replication, apoptosis and survival, angiogenesis, and much more. However, this modulation of the cellular miRNA pool is not uniform among all cell lines, viral strains, and infection types (lytic vs. latent). This may stress the need for further standardization of the experimental system.

Taking into account the various capabilities of CMV which are reviewed in this issue, as reflected by various profiling methods and through novel experimental models, we can now begin to fully appreciate the real extent and complexity of this virus. We can make use of these observations to deepen our understanding of immune activation in general and to design innovative targeted therapies, for example, antagomiRs, antiviral interventions, and HCMV-based vectors for the treatment of HCMV infections.

Acknowledgements Authors thank Alon Ziv for his generous contribution to the cover picture of this issue. 